\title{
The Right to Help and the Right Help: Fostering and Regulating Collective Action in a Medical Making Reaction to COVID-19
}

\author{
Megan Hofmann \\ Human Computer Interaction \\ Institute, Carnegie Mellon University \\ Pittsburgh, PA, USA \\ Rosa I. Arriaga \\ School of Interactive Computing, \\ Georgia Institute of Technology, \\ Atlanta, GA, USA
}

\author{
Udaya Lakshmi \\ School of Interactive Computing, \\ Georgia Institute of Technology, \\ Atlanta, GA, USA \\ Scott E. Hudson \\ Human Computer Interaction \\ Institute, Carnegie Mellon University \\ Pittsburgh, PA, USA
}

\author{
Kelly Mack \\ Paul G. Allen School of Computer \\ Science, University of Washington \\ Seattle, WA, USA \\ Jennifer Mankoff \\ Paul G. Allen School of Computer \\ Science, University of Washington \\ Seattle, WA, USA
}

\begin{abstract}
Medical making intersects opposing value systems of a medical "do no harm" ethos and makers' drive to innovate. Since March 2020, online maker communities have formed to design, manufacture, and distribute personal protective equipment (PPE) and other medical devices needed to fight the COVID-19 pandemic. We present a participant observation study of 14 maker communities, which have developed differing driving principles for efforts with varied access to interdisciplinary expertise on online platforms that mutually shape collective action. Over time, these communities unintentionally align towards action-oriented or regulated practices because they often lack higher level insight and agency in choosing communication platforms. In response, we recommend: regulatory bodies to build coalitions with makers, online platforms to give communities more control over the presentation of information, and repositories to balance needs to distribute information while limiting the spread of misinformation.
\end{abstract}

\section{KEYWORDS}

3D printing; collective action; COVID-19; making

\section{ACM Reference Format:}

Megan Hofmann, Udaya Lakshmi, Kelly Mack, Rosa I. Arriaga, Scott E. Hudson, and Jennifer Mankoff. 2021. The Right to Help and the Right Help: Fostering and Regulating Collective Action in a Medical Making Reaction to COVID-19. In CHI Conference on Human Factors in Computing Systems (CHI '21), May 8-13, 2021, Yokohama, fapan. ACM, New York, NY, USA, 13 pages. https://doi.org/10.1145/3411764.3445707

\section{INTRODUCTION}

Medical making, a practice of designing and crafting medical devices, has a long history of influencing healthcare practice [11]. In the last decade, advances in digital fabrication and online communities have produced a few notable initiatives (e.g., E-nable [36], Glia

Permission to make digital or hard copies of all or part of this work for personal or classroom use is granted without fee provided that copies are not made or distributed for profit or commercial advantage and that copies bear this notice and the full citation on the first page. Copyrights for components of this work owned by others than ACM must be honored. Abstracting with credit is permitted. To copy otherwise, or republish, to post on servers or to redistribute to lists, requires prior specific permission and/or a fee. Request permissions from permissions@acm.org.

CHI '21, May 8-13, 2021, Yokohama, Japan

(C) 2021 Association for Computing Machinery.

ACM ISBN 978-1-4503-8096-6/21/05 ..\$15.00

https://doi.org/10.1145/3411764.3445707
[38], MakerNurse [14], NightScout [33]). In the wake of the COVID19 pandemic, relatively rare medical making activities became a more global phenomenon. Expert-amateurs began to reconcile disrupted supply chains of critical medical supplies by 3D printing and sewing Personal Protective Equipment (PPE). We observed a fundamental transformation of medical making in reaction to: the disruption of regular medical device supplies, strict oversight of medical device regulation, and atypical social-distancing requirements in real-world interactions which pushed maker's further into online spaces.

To better understand these changes, we present a participantobservation study of online medical maker communities' reactions to COVID-19. In this study, conducted between March and June of 2020, we observed 14 online maker community efforts to make and distribute medical devices towards shortages in PPE. While multiple communities represent a global effort, our findings are grounded in the healthcare infrastructure of the United States. Our findings reveal how all 14 communities establish norms that effect the quality, sustainability, and impact of their efforts.

We present three themes synthesized from our study. First, we identify prevailing and sometimes conflicting ethea that guide these maker communities' action-oriented, safety-driven, and designdriven efforts. Second, we identify forms of expertise (i.e. principled knowledge, professional practice, embodied experience) valued by makers depending on access and clarity. Third, we identify ways that online communication platforms can inhibit community planning, access to established procedures, and productive engagements.

We describe maker communities on a spectrum between internally and externally regulated efforts. Makers self organize into communities guided by their personal ethos and develop new norms which prioritize specific forms of expertise. This can lead to communities positioning themselves on extremes of the spectrum between action and regulation, a trend which may be exacerbated by misaligned maker and medical values.

We make three recommendations which will enable maker communities to exert more control over their position on this spectrum. First, we argue for more clarity and outreach from regulatory bodies to foster a better relationship between makers and regulation. Second, we recommend that online platforms give community moderators more control over content presentation. Finally, we describe advances in repository infrastructure to promote rapid dispersal of 
safe and reliable designs while limiting the spread of outdated or unregulated information.

\subsection{Background: Making for the COVID-19 Pandemic}

Under normal circumstances, medical device manufacturing processes in the U.S. are overseen by regulatory bodies (e.g., Food and Drug Administration (FDA)) [19]. The liability for proper use of those medical devices is conferred onto medical practitioners under medical-malpractice law [29]. Regulation further varies across states.

The COVID-19 pandemic drastically increased the global demand for PPE while slowing critical supply chains. In response, the regulators began to lift testing restrictions for emergency-authorized PPE in February of 2020 [5]. In mid-April, the FDA issued an Emergency Use Authorization (EUA) to increase the production and distribution of these low-risk Class I devices [5]. This EUA lifted some but not all medical device distribution regulations. Some makers took this as the legal justification for them to manufacture and distribute PPE.

Within days of several states adopting stay at home orders, numerous makers posted open source designs for face shields, cloth masks, and respirators with significant variations in quality. Maker efforts attracted immediate and overwhelmingly positive media attention, which may have bootstrapped more initiatives [28]. In place of physical maker spaces, maker communities appropriated social media platforms to organize their efforts.

The NIH 3D Print Exchange, a repository used for collecting 3D-printable models related to biomedical science and healthcare [18], created a collection of COVID response medical designs. Submitting a design to this collection queued it for clinical review by the National Institute of Health (NIH) [34]. The collection is broken into four sub-sections: "Reviewed for Clinical Use" which implies that the designs are likely safe in a clinical environment, but does not imply traditional device approvals; "Designs Optimized for Community Use," which are not necessarily found to be safe in a clinical environment, but safe in community environments such as grocery stores (i.e. the device will not hurt you but it may not prevent the spread of COVID-19), "Warnings" which denote designs that are not verified as safe or require further FDA approvals, and a catch-all set of prototypes which have not yet been reviewed.

\section{RELATED WORK}

In this section we outline the norms and motivations of makers described in the literature, the influence social media has had on other forms of crisis response, and how rapidly growing online communities actively establish norms.

\subsection{Making and Healthcare}

Past studies have shown that, generally, maker culture values innovation, openness, and altruism. Lindtner et al's ethnographic studies show how makerspaces are established as sites of innovation [26, 27], Tanenbaum et al's review of making as democratizing technology shows that maker culture tends to favor particular "hedonized technologies" (e.g., 3D printers), which are exciting to work with or help the maker establish credibility in the community [45], over other crafting approaches. Outside of these physical spaces, there are many open-sharing platforms used by makers (e.g., Thingiverse [3], Instructables [40], GitHub [7]). Kuznetsov and Paulos's survey of makers in a range of these communities demonstrated a core value of information exchange where makers are expected to share intermediate artifacts to contribute to collective sense making [23]. Alternatively, when makers associate their practice with care-work beyond efforts to innovate and learn from others, some makers are intrinsically motivated to help [48] and extrinsically motivated by communal recognition for doing good [13]. Vyas's interviews of women makers showed a relationship between maker communities that emphasize care and the values of the makers themselves [48]. Fox et al. extended this understanding by evaluating the way in which makers in a feminist hacker space use making as a tool to work out their place in that community and their relationships supporting others. Buehler et al. and Parry-Hill et al. showed a similar correlation between makers of assistive technology and communities of disabled people and care givers $[3,36]$.

However, it remains unclear how values of openness, and altruism are managed in medical domains where the risks associated with innovation and openness may conflict with ideals of "doing good" [15]. While similar in scale to that of hobbyist and entrepreneurial making, medical making carries greater risk. When clinical staff make, they act out of an ethos to "do no harm" $[15,16,24,41]$ because patients are vulnerable to the most risk $[8,35]$. In order to mitigate these risks when studying medical making, researchers have either opted to study clinicians while avoiding delivering a product to patients (e.g., [15, 31, 41]) or to use a researcher's fabrication capabilities to move the locus of responsibility onto the researcher (e.g., $[4,16])$. When medical making has been studied in the wild [24, 30,51], we find clinicians tend to assume responsibility for its risks by keeping the whole design at the point-of-care [24] rather than openly sharing the process. Here, an open source model of making is incompatible with the realities of the medical field because the open exchange of intermediate artifacts would expose incomplete and possibly dangerous designs to the public.

\subsection{Social Media and Conspiracies During Crisis}

The role of online communities in crisis response depends on the communities' perception of institutional authorities. On the one hand, Utani et al's survey of counter-disaster systems developed by Japanese makers, argues that government officials could effectively coordinate with online communities if they rapidly deployed relevant data [47]. On the other hand, Evans et al's examination of the formation of social media based solidarity economies in Venezuela demonstrates these communities' desire to remain separate and unregulated by the government which they viewed as contributing to the crisis [10]. Pre-existing relationships between the constituents of online communities and the relevant authorities (e.g., governments) may influence the communities' effective response [1].

Particularly during crisis, conspiracy theories can spread through online communities when amplified by trusted community members and the media. In response to crisis, Kou et al's examination of 


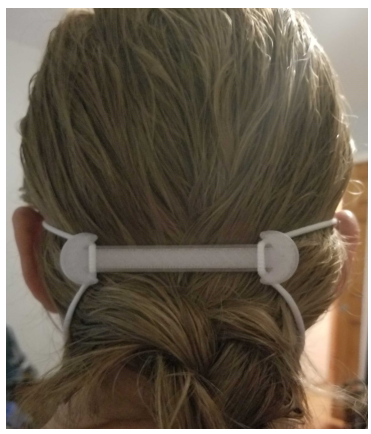

(a) Ear-Saver Mask Accessory

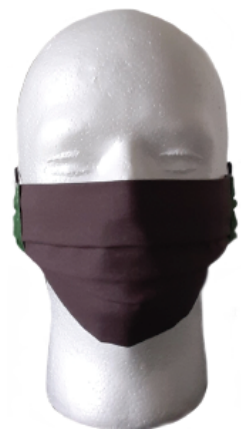

(b) Sewn-Cloth Face Covering

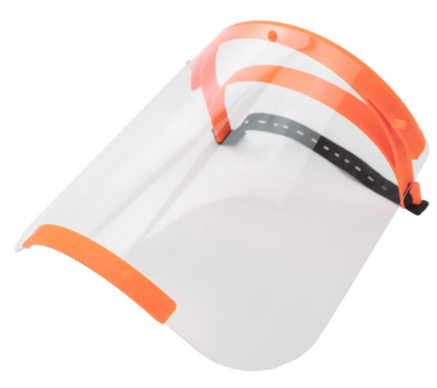

(c) Face Shield

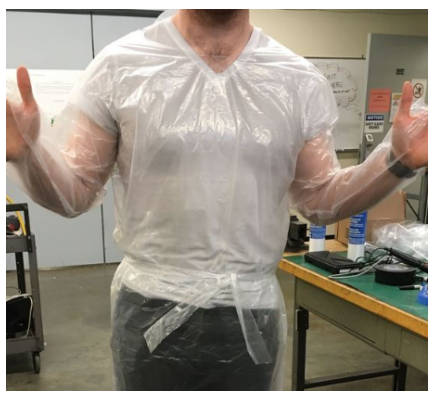

(d) Disposable Protective Gown

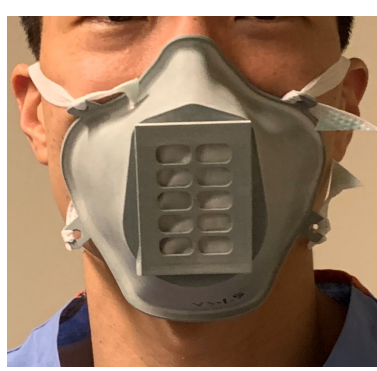

(e) N95-Style Face Mask or Respirator (2).jpg

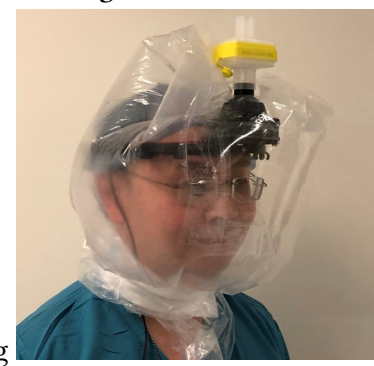

(f) Personal Air Purification Respirator (PAPR)

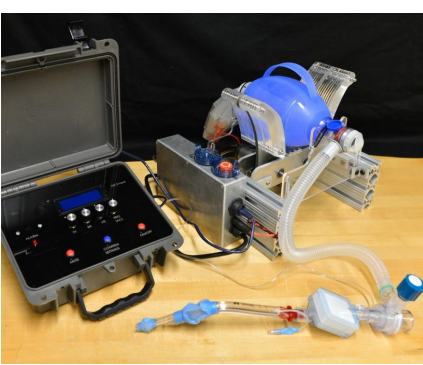

(g) Ventilator from https://e-vent.mit.edu/

Figure 1: Sample maker PPE presented in ascending order of risk from least to greatest risk based on FDA medical device classifications. Makers produced a wide range of medical devices, primarily PPE (a through f). All but (a) the cloth mask and (f) the ventilator were collected form the NIH 3D Print Exchange. The cloth mask is from an online maker community website and the ventilator design is by researchers at MIT.

information spread about the Zika virus demonstrates that people engage with conspiracy theorizing as a form of collective sense making. Wong-Villacres et al. 's study of the social media response to a 2016 earthquake in Ecuador shows that social media enabled unprecedented collective action but also spread misinformation too quickly for organizers to manage it [50]. Starbird et al's case studies of online information operations reveal an online ecosystem for conspiracy theorizing which influences adoption of conspiracy at its periphery [42]. Huang et al's case study on the 2013 Boston Marathon Bombings points to a higher susceptibility of misinformation spread among users who feel emotional proximity to the crisis space [17]. Regardless of misinformation's original source, Starbird et al's analysis of misinformation spread on Twitter showed that some power users (e.g., journalists) contribute to the early spread and acceptance of rumors [43]. The influence of journalists is particularly concerning when considering how media valorizes maker efforts [2, 28] without considering critical views (e.g., [13, 37, 49]). In the context of crisis, the spread of misinformation may impact how online communities take collective action or collaborate (or don't) with entities indicted by such conspiracies.

Online information exchange during crisis response is complicated by breakdowns in societal trust, authority or structure, and sustainable action. During wartime, Semaan and Mark's work indicates societal trust shifts from institutions to new information spaces where members articulate their response to a crisis [39]. Torrey et al's review of online "connected giving" groups for the
2007 Hurricane Katrina reported smaller blog communities evolved a centralized structure with high trust and low sustainability unlike decentralized forums [46]. Auferbauer and Tellioğlu locate a difference between formal and informal responses to the 2015 European migration crisis in members' organizational backgrounds [1]. Informal "spontaneous volunteers" take ad hoc self-organized approaches to online action rejecting established rules and processes in favor of internal flexibility. Sutton et al. explain the preference for self-supporting information sources with increased loss of trust in government and media in relief efforts for Southern California Wildfires [44].

\subsection{Norm Establishment in Growing Online Communities}

Some challenges faced by pre-existing online communities are: norm formation [12, 22], norm adherence and enforcement [21, 22], and information overload [20]. Fiesler and Bruckman's study of one online community demonstrated that norms in existing communities largely evolve from members observing and mimicking each other [12]. In the case of broad communities (e.g., Facebook [32]), where norms are not established, they derive from the participants external observations. Once such norms have been established, communities must protect their culture through socialization of new members [22]. As in previously studied discursive communities on Reddit [9], we expect this work is primarily managed by community 
moderators who, when facing a surge in conflict introduced by new members, are burdened with significant emotional labor. Jones et al's survey of Usenet news groups revealed that as communities grow, members and moderators tended to become overloaded with information [20]. In the context of medical making, this may lead makers to fall back on their personal biases towards innovation [27] and away from safety norms [15, 24]. Kiene et al's qualitative analysis of a similar surge (i.e., "eternal september") showed that managing these challenges required extensive labor from organizers [21]-a resource significantly limited when urgently reacting to a global crisis.

\section{METHODS}

We undertook a participant observation study of medical maker communities during the acute phase of the US response to the COVID-19 pandemic between March and June 2020. We observed public social media posts on 13 online maker communities while A3 observed and participated in a local site with insignificant social media presence, S2: a state university initiative across multiple departments (e.g., engineering, computer science, design, and hospital network).

\subsection{Participation, Observation, and Analysis}

We conducted this study in two overlapping stages: community identification and engagement. First, we connected to maker communities through our personal networks and analyzed public online content as digital traces of communities on Facebook, Reddit, and Twitter. A1 was an active poster on S3, S9, and S10's forums, and was included as an organizer in S1's community, A2 remained a silent observer of sites S3-S14. By separating these roles we can triangulate [6] our experiences as participants and observers.

We collected publicly available media articles, social media mentions, and documentation from these sites. Each researcher wrote memos multiple times a week, depending on community activity, documenting their experiences and impressions. The majority of the collected data are direct quotes of community posts and our retrospective analysis of them. However, we also include a few participant observations where they are particularly salient to emergent themes. Each week, researchers met to discuss their observations and activities during debriefing sessions as described by Lincoln and Guba [25]. In these sessions, we each described our weekly research activities and observations. Then other researchers critiqued and interrogated each others' findings. Notes from these sessions are used to develop themes which are then applied individually by researchers to their notes and memos. We repeated this iterative, inductive synthesis process throughout the study. We reached consensus on the presented themes of established ethos, accepted expertise, and platform limitations when multiple weeks of observations no longer raised new themes or discussion concepts.

We attempt to protect the anonymity of makers by: deidentifying their posts in our notes, quoting from the largest relevant communities, and modifying the specific phrasing enough to make posts unsearchable. Organizers from S1 and S2 provided informed consent for researchers to participate and observe the communities. These methods were approved by each author's university's Internal Review Board.

\subsection{Overview of Maker Communities}

We selected sites S3-S14 based on their prevalence on four media platforms (e.g., Facebook, Reddit, Slack, Twitter) (Table 1). We identified the largest community, S3, from mentions in media articles. Then, we searched for the terms PPE, COVID, Do-It-Yourself PPE, and masks to expand the community set. Starting in mid-March, we tracked their daily online activities to gather ephemeral data typical of quick response research [44]. The communities met three requirements; they must (1) be open to the public, (2) involve medical making of PPE during the COVID-19 pandemic, and (3) were active for the duration of the acute phase of the pandemic (i.e., March to June 2020). The resulting 13 communities cover a range of active, public, and online maker communities reacting to the pandemic during these months. All communities applied both hightech digital fabrication technologies (e.g., 3D printing) and low-tech crafting technologies (e.g., sewing) to manufacture medical devices. Quoted threads (e.g., Th1) and makers (e.g., M1) are numbered in the order they are presented in the paper.

While a few communities represent global organizations (e.g., S3S6), the majority of communities operated primarily in the United States. Additionally, all observed online activities were conducted in English. As such, the majority of observed medical maker activities are effected by the institutional and regulatory practices of the United States.

For each community we have different levels of insights into their goals and organization structure depending on what is publicly posted. For example, the largest community, S3, provides a public facing charter document which we can analyse to infer their organizational structure and goals. Generally, our observations are limited by the ongoing, always on nature of online discussions. We are unable to present evidence of posts that were either deleted or edited by participants. In effect, if we did not observe the conflict in real time, it was likely not to have persisted on the forum.

\section{FINDINGS}

Based on the surge of medical making for the pandemic, our goal was to observe interactions between stakeholders to understand emerging norms which effect the impact of these efforts. We summarize our findings as follows:

Established Ethos: Community discussion over norms determined trade-offs between: action, safety, and design innovation.

Accepted Forms of Expertise: Communities accepted expertise expressed as: principled knowledge; professional practice; and embodied experiences.

The Limitations of Community Organization Platforms: While online platforms enabled the unprecedented maker community organizing needed to take action, features of these platforms also disrupted community planning, hid procedures and content, and fostered destructive engagement and conspiracy theories.

\subsection{Establishing Ethos}

We observed that many of these communities work to establish a shared ethos explicitly through community charters or documents, or implicitly through the language and actions of vocal members. Larger sites (e.g., S3) tended to self-align based on their explicit approaches to norm formation. We identified three core values 
Table 1: Observed Sites and online communities described in terms of: locality (e.g., city, state, national, global), PPE products, online platforms, Organization legal structure, and membership size (e.g., less than 100, between 100 and 500 , between 500 and 1000, between 1000 and 5000, between 5000 and 10,000, between 10,000 and 50,000, and greater than 50,000).

\begin{tabular}{|c|c|c|c|c|}
\hline ID & Locality & PPE Products & Platforms & Size \\
\hline S1 & State & cloth masks, ear savers, face shields & Facebook, Mighty Networks, Slack, website & $<5000$ \\
\hline S2 & City & face shields, gowns, respirators & Slack, website & $<100$ \\
\hline S3 & Global & cloth masks, ear savers, face shields, respirators, ventilators & Facebook, website, Slack & $>50,000$ \\
\hline S4 & Global & cloth masks, ear savers, face shields & Facebook, website, Twitter & $<50,000$ \\
\hline S5 & Global & cloth masks, ear savers, face shields & Facebook, website & $<5000$ \\
\hline S6 & Global & cloth masks, face shields & Facebook, website, Twitter & $<5000$ \\
\hline S7 & National & cloth masks, face shields, respirators, ventilators & Facebook, website, Twitter & $<5,000$ \\
\hline S8 & National & ventilator & Facebook & $<500$ \\
\hline S9 & State & face shields & Facebook, Slack & $<1000$ \\
\hline S10 & State & cloth masks, face shields, respirators & Facebook, website & $<1000$ \\
\hline S11 & City & cloth masks, ear savers & Facebook, website, Twitter & $<10,000$ \\
\hline S12 & City & cloth masks, ear savers & Facebook, website, Twitter & $<5000$ \\
\hline S13 & City & cloth masks, ear savers, face shields & Facebook, website, Twitter & $<1000$ \\
\hline S14 & City & cloth masks, ear savers, face shields & Facebook, website, Twitter & $<500$ \\
\hline
\end{tabular}

across these maker communities: action-oriented, safety-driven, and design-driven communities. While these values are not always in conflict, makers frequently treated them as antithetical.

4.1.1 Action-Oriented. An action-oriented ethos is largely owed to the urgency of the pandemic crisis. These communities quickly recognized that collapsing supply chains would result in significant loss of life. In turn, makers reacted with a feeling of moral responsibility to take action. Respectively, any actions that slow down that response were seen as immoral. In some cases, the perceived binary between do-ers and obstructionists was expressed with vitriol.

S3, S4, S11, and S12 had periodic discussions about convincing local hospitals to accept makers' PPE. Due to inconsistent regulation and variance in local needs, facilities had different standards. Some refused non-traditional PPE; others accepted some clinically reviewed designs and procedures, while many took whatever they could find. Rejected makers within communities (e.g., S3, S4) expressed their frustration and sought action-oriented solutions to circumvent administrative policies, such as donating to less risky facilities or arguing to circumvent administrators by delivering PPE directly to practitioners:

"Ask for forgiveness rather than permission. Less thinking and more action, go directly to the doctors and nurses who don't have any better alternatives. Contact the hospital directly and you will get administrators and executives. Ignore them." (S3, Th1, M1)

Many members of these communities (S3, S4, S10, S12, S14) saw actions taken to slow down the group, even in the pursuit of safety, as unethical. Their core argument was that the groups served a critical purpose where something is better than nothing. For example, S3 called themselves a "do-ocracy," where members take immediate action and should only be obstructed when another active member raises "serious concerns" by taking a counter action. This forced the community to constantly take action.
Makers tended to prioritize material efforts. Within S3, if a member raised concerns but could not demonstrate material, tangible participation, their perspective was devalued. For example, A1 identified herself as a researcher and asked a maker to clarify their "clinical approval". After criticising A1's association with academia, he continued to dismiss her contribution as an organizer. He belittled the in-material work of research and organizing by asking her for evidence of her tangible efforts:

"Not to be mean, but how much PPE have you personally made?" (S3, Th2, M2)

A1's perceived obstructive actions were seen as dangerous. M2's view that slowing down was unethical, to him, justified the use of violent language. We suspect that similar violent discourse caused many makers to not voice regulatory views in these communities, or leave all together. Based on an overwhelming negative reaction to A1's request she stopped replying to Th2. However, M2 added eight posts with escalating violent language ending with:

"If my masks are so dangerous we should force you to wear one so you get COVID and die, you [expletives]."

$$
\text { (S3, Th2, M2) }
$$

While a bias towards action angered and emotionally charged discussions with a vocal minority of makers in some communities (S3, S4, S11, S12) in others (S1, S2, S5, S6, S7, S13) it motivated strategic and multilateral organization. In a thread discussing S1's mission, one maker posted a document they prepared outlining a guide for stop-gap maker PPE efforts. In it, she highlights the relationship between organization and working quickly:

"Get organized! I have seen many teams running with the Maker Efforts and delivering successfully. The roles

in each team varies with the quick pace that was

needed for teams in this call to action." (S1, Th3, M3)

4.1.2 Safety-Focused. Many communities retained a medical ethos of "do no harm," which emphasizes caution, safety, and evaluation. 
Safety-focused communities saw infection risks as a significant barrier; if any makers were infected because of their making activities then that would diminish the good done by the community. In response they started by minimizing risk, then maximizing good. Within these communities, safety became the basis for most community norms, and the safety and regulatory experts emerge as community leaders.

Safety evaluations were applied to all of S1's procedures. Their "safety team", led by two medical researchers, produced Standard Operating Procedures (SOP) which explained how to do tasks safely by minimizing the risk of contracting and spreading the virus. There were SOPs for every task in the community (e.g., making, deliveries, warehouse management). M4, a safety lead, explained this approach during a publicly streamed safety question and answer session:

"When we designed the SOP, we built in multiple points of redundancy to allow for the possibility that every maker would not do things correct every single time, rather than relying on a single step where, if it failed, the entire thing would go pear shaped." (S1, Th4, M4)

Other communities (S2, S5, S8, S9, S13,) also followed this approach, but with varying degrees of enforcement. S9 established community values of social distancing and cleanliness. However, they had no public documentation of these procedures which caused some confusion. A1 asked on their Facebook Group for any documentation and a core organizer responded with a brief procedure description. They could not provide any other documentation:

\section{"Once the shields are ready, we have the drivers schedule pick up and drop them all off at the warehouse for sanitation. And then the doctor liaison directs the distributions." (S9, Th5, M5)}

Safety dictates these communities' design and production processes. S2 has a safety-focused design approach. They designed and evaluated a 3D printed respirator which passed NIH 3D Print Exchange's clinical review after 13 days of development. Rather than sacrificing safety evaluations, the speedy design process came at the cost of make-ability. The design is printed with non-consumer SLS 3D printers, and cannot be readily produced by hobbyist communities. Using manufacturing processes that were known to be safe (i.e. easy to disinfect) sped up the design process. However, distributing the design to public communities remains a significant challenge since that level of safety cannot be assured. S3 makers mentioned S2's design as an example of a safe design and asked how to print it in hobbyist 3D printing filaments, implying they did not recognize the requirement to print with non-consumer machines to ensure safety. Ultimately, S3 disregarded the design because they did not have the resources to manufacture it.

4.1.3 Design-Driven. To an extent, every maker community we observed was driven to innovate through design. Each community used and disseminated their own designs and procedures, which they either created internally or remixed from other communities' content. While some communities (e.g., S1, S9) remixed designs to fit their specific community needs, others (e.g., S2, S3) prioritized creating new designs. Almost all communities had to innovate on community procedures to make them fit their design needs.
Design-driven communities distinguished themselves from action-oriented communities by prioritizing designs over production outcomes. While action-oriented communities (e.g., S4, S9, S10, S11, S12) aimed for impact by supplying their local community, design-driven communities (e.g., S2, S5, S8, S13) desired the impact of releasing a design beyond their own community. Many communities pursued both by segmenting into design and production efforts, however this solution occasionally caused conflicts as limited resources were diverted to either design or action. For example, S2 primarily focused on creating innovative and safety regulated designs, but also printed face shields for its medical facilities. Both projects required $3 \mathrm{D}$ printers, and $\mathrm{A} 3$ was almost always told to prioritize printing a part for prototyping over printing more PPE. Additionally, S2's administrators refused efforts to distribute PPE outside the university because they determined this was not an acceptable use of grant funding. From their perspective, design is research, appropriate for grant funding, but external engagement is not. Their designs were shared on the NIH 3D Print Exchange, but without partnership with communities who manufacture and deliver this PPE it is unclear if the designs will have significant external impact.

Amongst these communities, design innovation was viewed as a moral responsibility. Some communities (S3, S8, S13) prioritized challenges when deciding what to design. One of the clearest examples of this was efforts to create DIY ventilator designs, despite debates about the morality of pursuing such high-risk devices:
"There is an argument that something is always better than nothing, and that if you're literally suffocating, you'll take your chances. But the more I understand about ventilators, the more I fear building them. I have no interest in making a machine that is going to kill people." (S3, Th6, M6)

Many makers on S3 announced their support of this warning, remarking that it laid out the risks in a useful and clear way. While it was a heated debate, the majority of makers who responded to the post disagreed that the risks were sufficient to stop designing alternatives. The makers reached a consensus that, since people would presumably die without a ventilator, there was no added risk to patients. In a sense, makers believed they were not responsible, nor were the medical practitioners who would use their ventilators. The most popular rebuttal, based on likes, was:

"It is time to let go of old ways. Yes, the ventilator will probably kill some people. But what is the alternative? The question is, let people die for lack of a multi-thousand dollar ventilator? Or risk saving a few with ours while we perfect it. In a perfect world the manufacturers would have published their specs. But this is an imperfect world, ruled by greed. Which side will you be on six months from now?" (S3, Th6, M7)

Some makers in design-driven communities engaged in conspiracies that medical device regulation is an intentional effort to slow down open source efforts. Within threads across larger communities (S3), smaller groups exchanged these ideas in favor of specific manufacturers, halting their innovation. For example, M8 argued:

"In this emergency the face shield is a lifesaver and any stalling by way of 'approval' is only about the 
money and mates-rates quangos [sic]. Unfortunately

by comparison, companies are fast-tracked without such lengthy 'approval'.” (S3, Th7, M8)

Overall, design-driven communities distinguished their efforts from action-oriented communities because collective action drained design resources. A3 witnessed this in the gown prototyping process. After days of prototyping, part of the team wanted to start user-testing (i.e. action-oriented) and others wanted to continue prototyping (i.e. design-driven). In a community that has room for both perspectives, this led to a healthy dialogue. In others, safety was considered a valued goal but not when regulation in the form of "lengthy" clinical approval would obstruct efforts. Action oriented communities valued design and safety only when it served taking action. Safety driven communities ultimately desired to design and take action, but would forgo activities experts deemed unsafe.

\subsection{Accepted Forms of Expertise}

To justify their positions, makers appealed to various forms of expertise. Across these communities, we observed three accepted forms of expertise: principled knowledge, professional practice, and embodied experience. Principled knowledge was derived from institutions that primarily produce knowledge such as academia and government agencies. Expertise derived from professional practice was the expertise of front line healthcare workers (e.g., doctors, nurses) who use PPE. Finally, makers appealed to the expertise they achieved from their embodied experiences while making.

4.2.1 Principled Knowledge. Under normal circumstances, principled knowledge is disseminated through peer-reviewed publications and government agencies. By principled knowledge, we mean the generalized knowledge that has been produced and reviewed by institutionally-recognized experts to develop disciplinary consensus which is then disseminated to the public. Many makers across a cross-section of ethos in communities (e.g., S1, S2, S11, S13) viewed this knowledge as the gold standard for guiding design processes and organizing their efforts. For example, M9 asked for peer-reviewed research and received seven citations in the comments:

\section{"I'm looking for the best scientific paper on filter materials. Comments are desired." (S3, Th8, M9)}

Principled knowledge about COVID-19 was being generated and disseminated unusually quickly as society attempted to manage the pandemic. Maker communities paid significant attention and scrutinized interpretations at length. Makers in some communities (e.g., S1, S4, S12) viewed unmitigated discourse amongst experts for the first time and became aware of the constant debate over various sources of new information. This led to conflicts and frustration as makers share new knowledge that appears to conflict with the sources cited by other makers. Another maker in S3 requested peerreviewed research on related topics. The ensuing discussion traced a heated debate which compared a number of highly technical details such as the electrostatic charge of the virus and commercially available respirator filters. An active commentator became frustrated, notable in the deterioration of spelling, after repeated defense of their insights on filtration efficacy of a chosen material.

\author{
"Read my above statement filter material. Then go to \\ my example and read the messages. I used that \\ example to make a point. Been doing this for a while. \\ Your info that you provided is good but there are new \\ people trying same thing." (S3, Th9, M10)
}

At times, principled knowledge was disseminated too late, which created conflicts between best practice and current practice. For example, $\mathrm{S} 1$ began producing face shields before the establishment of the NIH 3D Print Exchange. S1 had consistently espoused a core value of safety and clinically reviewed all of their designs. However, after some face shield designs received the NIH 3D Print Exchange clinical review, makers who were not included in those initial testing phases became confused between conflicting messages from government and the S1 leadership. During a public quality control town hall, one maker, M11, asked why the community would not accept the face shields he had printed that the NIH had " $a c$ cepted". The moderator explained the S1 clinical review process and that M11's design was not compatible with the community's laser cut shields. M11 seemed satisfied and switched to the community design. The temporal conflict between the principled knowledge disseminated by the NIH 3D Print Exchange and S1 leadership, confused M11 until he had insights into the community's internal decision making process.

While M11 accepted this conflict, others (e.g., S3, S7, S12) saw academics and government officials as untrustworthy and corrupt. M2 perceived A1's request for information about his design as criticism and asked her to justify her comments. She re-identified herself as a researcher and provided references to her past work. Providing her publications was an appeal to her principled knowledge. M2 then expressed his distrust of academia and research:

\section{"Published? I have little respect for that. That has not helped. You just glad-hand politicians and academics while taking credit makers' work." (S3, Th2, M2)}

4.2.2 Professional Practice. In tandem, and sometimes supplementary, to principled knowledge, professional practitioners were viewed as critical experts in these maker communities (e.g., S4, S6, S11, S12). Professional practice refers to people who work in a field relevant to responding to the pandemic, primarily medical practice. Some makers viewed professional practice as a substitute for, or improvement on, principled knowledge. Practitioners are considered experts because of their experience applying relevant knowledge to medical practice, and because they are the primary users of PPE.

In some cases (e.g., S4, S10, S12), makers believed professional practice should override official guidelines. For example, "approval" from medical practitioners was frequently cited to justify design choices, even when this contradicted $\mathrm{NIH}$ guidelines. A newspaper covered one maker mask as the first "federally approved mask of its kind", only once noting that the NIH 3D Print Exchange had not found the design to be safe for clinical use. However, the article contradicted this assessment by regarding a local hospital doctor who had user tested the design. Makers, such as M12, weighted the expertise of the practicing doctor over the NIH's guidelines:

"Perhaps everyone printing respirators should switch to this mask?" (S10, Th10, M12) 
Some communities evaluated their designs with medical practitioners (e.g., S1, S2). For example, S2 conducted multiple rounds of user testing with medical practitioners. A3 noted that the prototyping team would reach a point in the design process where they could not innovate further without feedback from those who would be using them everyday.

Within other communities (e.g., S3, S4, S7, S11, S12), makers appealed to hospital "culture" to justify decisions. A resolution emerged deep into Th11, debating various mask filter materials, based on makers' nuanced observations of the negotiation between hospital needs and maker responsibilities. They described the challenges of using "technical things" and "studies" (i.e. principled knowledge) amidst shifting consensus in the scientific community. They appealed instead to the preferences of local practitioners and note how physical efforts to produce and distribute masks will be more practically valuable in the "desperate situation." In this way, M13 appeals to practitioner's expertise to assess needs:

"People are treating filter materials as a "technical"
thing. It's partly that, but it's also the culture of the
hospital and the people who use it. We point them to
our resources so they can decide on their own. If they're
fearful, 100 studies won't convince them, and if they're
desperate, they may not need studies." (S3, Th11, M13)

Among cloth mask makers, a similar trend extended to personal networks. S12 opened a debate about "medical grade cloth" by requesting guidance from medical practitioners. Cited practitioner expertise ranged from midwives forums to conversations with medical-professional spouses. The maker abandoned efforts to source the material reverting to recycling cloth.

\section{"This isn't available to us regular Shlubs. \\ Manufacturers need it more. I'm using old bed sheets."} (S12, Th12, M14)

4.2.3 Embodied Experience. Finally, many makers (e.g., S1, S3, S5, $\mathrm{S} 8, \mathrm{~S} 13$ ) valued the expertise derived from hands-on practice (i.e. embodied experiences). Practice may not make perfect but it does generate expertise; makers learned by doing (e.g., 3D printing, sewing, engaging in forums, organizing). The expertise gained through action elevated individual makers within the community. This expertise was demonstrated best in the continuous skill-share between experienced and novice makers. For example, S13 opened up membership to support makers outside their local community. A novice maker from another country posted asking for help. Experienced community members shared their expertise and guided the novice over six weeks, step-by-step, during their initial attempts to print masks with new materials.

\section{"Massive fail tonight! Every mask failed. I have two printers and lots of PLA and time. If anyone has a file they can share that would be great. I have zero skills. Thanks in advance." (S13, Th13, Th14)}

Within S1, shared embodied experiences of making elevated particular makers to positions of greater influence. M15 organized 3D printing help calls most nights during the peak production period. These calls were initially intended to support makers who were struggling to manage their printers. His embodied experience using a variety of different consumer printers helped him coalesce a sub-community of 3D printing experts. Other experienced makers emerged in this group and supported others. This manifested an ongoing virtual skill exchange. Additionally, these calls served as an engaging, almost therapeutic, environment for makers to share experiences and recognize each others contributions.

Communities valued forms of expertise based on their access to information and their collective sense-making abilities. Communities that had academics and medical professionals as members tended to favor principled knowledge, even when sources offered conflicting insights, and the advice of practitioners. Communities whose leadership was dominated by active makers and hobbyists tended to favor skill-exchange based on embodied experiences.

\subsection{Platform Limitations on Organization: Planning, Procedure, and Engagement}

Social distancing requirements forced these communities to organize remotely, rather than physically interacting with each other. Due to a sudden reaction to the crisis all of these communities seem to have selected collaboration platforms based on their founders' familiarity and availability. Facebook Groups and Slack were the two primary communication platforms in the communities we observed. These platforms significantly shaped communities and sometimes contributed to social breakdowns that we categorize as: disrupted planning, lost procedure, and destructive engagement.

4.3.1 Disrupted Planning. Organizers developed plans and procedures for community activities, but, occasionally, the structure of Facebook Groups and Slack disrupted these efforts. Organizational efforts were sometimes lost or done in parallel leading to conflicts later on. Despite initial attempts to organize efforts on Facebook, several communities additionally relied on external tools (e.g., websites, email, phone, Slack). Occasionally, posters would request additional volunteer labor, data (e.g., PPE counts) from the community or offer a collective resource. In the limited contexts where we had visibility (S1, S2, S9, S11, S12), these requests were usually met with requests for private conversations about organizing activities.

Unlike the generalized features of Facebook Groups, Slack is designed to support organizational efforts. Despite this purpose, seemingly important discussions and threads were often overlooked when posted in "\#general" channels. For example, one maker, M16 asked the S9 Slack how to report his COVID infection and have his face shields pulled from the supply chain. The post received numerous reactions (e.g., thumbs up) but no replies. M16 re-posted the question multiple times, reiterating his concern that he had introduced COVID-19 into the supply chain. To date, we have not found a S9 policy for tracking the spread and we did not find any public conversations about how to introduce a tracking protocol despite apparent interest from the makers.

4.3.2 Lost Procedure. The key artifacts produced by organizers are community procedures that ensure community members are aware of and follow established norms, work safely and effectively, and produce work that can be distributed by the community. This necessitates that community members find and access these procedures before taking actions that conflict with them.

Several online communities (e.g., S3, S9, S11, S12, S13) pinned their procedural documents in Facebook Groups at some period 
of time. However, only one document or post can be pinned at a time creating additional labor for organizers. The S9 community stored on-boarding information in a FAQ document linked in the files tab on their Facebook Group's main page. When first entering the group, there was no indicating that the document exists unless another maker recently linked to it. As a result, many new members repeatedly asked the frequently asked questions causing moderators to send them to the information. One of these moderator's responses reveals their frustration with Facebook and a conflicting feeling of necessity since Facebook is a ubiquitous tool which can connect needed volunteers to the community:

$$
\begin{gathered}
\text { "We're constantly trying to revise the FAQ for } \\
\text { newcomers. Unfortunately, Facebook was the easiest } \\
\text { way to get the word out." (S9, Th15, M17) }
\end{gathered}
$$

While Slack can be more easily integrated with file management and repository tools (e.g., Google Drive, GitHub), pointing makers to stabilized procedural information remained a challenge. In Slack, content tended to get siloed across different channels and threads. For example, in gown prototyping, the $\mathrm{S} 2$ team struggled to develop a system where designers could leave the files for the manufacturers to find and produce them. The number of pins, linked documents, threads, and tags directed at particular team members was overwhelming. Over the course of two days, outdated design documents were used with incorrect gown-assembly procedures because information was missed in a particular thread. At times, other sources of confusion were passed on to the medical professionals who received the PPE. Without a clear chain of documentation, some practitioners were under the impression that the face shields were disposable like traditional face shields. So they threw them away rather than cleaning them, a tremendous loss for the makers. This ill-supported information exchange ended in frustrated team dynamics, lost physical resources, and wasted time.

4.3.3 Destructive Engagement. Many communities, particularly those that relied solely on Facebook (e.g., S4-S8, S11, S12), struggled to manage community discussions that contained disinformation, and, at times malicious discourse. Community members sometimes propagated, maliciously or unintentionally, disinformation informing community practice. The platform infrastructure increased spread and organizers struggled to limit disinformation.

Within some maker communities (e.g., S3, S4, S12), disinformation derived mostly from conspiracies that government regulators were working with medical device companies against makers and open source designers to ensure their monopoly, despite the desperate need for increased PPE supplies. Even when some makers attempted to refute this belief, the refutation could, at times, propagate different forms of disinformation. For example, a maker, M18, shared a fake-news article about two men who had designed a 3D printable version of a ventilator valve. The article falsely claimed that the men were sued by the manufacturer, causing the design to not reach hospitals that requested the part. M18 notes that the article has been debunked, but then falsely claims that the disinformation had been spread by the government to cover up the fact that regulators had prevented the manufacturer from releasing the valve design. In fact, the articles M18 cites debunking the original article explain that hospitals had rejected the valves because they did not meet the same quality standards as the traditional parts. Neither corporate nor government conspiracy was involved. In an attempt to correct disinformation, M18 propagated conspiracy theories:

"Pure propaganda. I'll leave it to you to figure out what sort of 'people' might run such a story to gain political points at a time like this...The reason the manufacturer couldn't release the design was because of government medical manufacturing regulations." (S3, Th16, M18)

Posts containing this conspiratorial talk tended to attract significant engagement in the form of likes and comments, which may have increased the likelihood to be presented to other members which in turn could increase the engagement further. As the threads get longer and harder to follow, members were more likely to express their frustration through name calling. In Th6, where M6 argues against designing DIY ventilators, M19 accuses the original maker of being an "operative" for the medical device companies trying to dissuade the community from competing with these companies. More makers then accuse M6 and the thread devolves to name calling.

$$
\begin{gathered}
\text { "Operative [M6] works for one of those money making } \\
\text { companies...I know this world too well. Everyone is } \\
\text { profiteering. Watch your back." (S3, Th6, M19) }
\end{gathered}
$$

While we did not observe similar purposeful and extensive spread of disinformation and conspiracy on Slack, confusion and information overload did, occasionally, spread disinformation. For example, makers would post questions in a S1 "safety" channel. These questions were directed at the safety leads, but sometimes questions got lost amongst discussions about the SOPs. Other makers would respond with answers when the safety leads were not available. However, there was no way of ensuring that the makers' responses were accurate and consistent with the SOPs. For example, in early April when the original safety channel was most active, one organizer, M20, asked the channel where to find updated SOPs. Another maker who was not a member of the safety team, M21, replied within 10 minutes to provide an older copy of the file which M20 then posted to the public making page. 10 minutes after that, one of the safety leads, M4, responded and tagged M21, but not M20, to clarify that an updated version needed to be used. It was not until a week later that the error was rediscovered and M20 corrected it on the public facing website. It is unclear what errors this introduced in maker practices during that time. In response to such miscommunications, M4 created a "safety-questions" channel, with an explicit rule that only the safety leads could provide answers.

\section{DISCUSSION: MODELS OF MEDICAL MAKING}

Medical maker communities exist across a spectrum of internallyand externally-regulated efforts. In the context of the COVID-19 pandemic, we identified themes describing how makers adapt a communal ethos, develop operational norms, and prioritize various forms of expertise to shape their practice of medical making. Through this analysis, we contribute insights into the social and technical aspects which shaped the development of maker communities participating in relief efforts and the impact of disinformation on these communities. On one side of this spectrum, action-oriented, 
grassroots maker communities function as internally-governing entities committed to producing material outcomes. At the other end, maker communities operate within hierarchical, externallyregulated environments and prioritize clinically reviewed standards. No single community represents an extreme end of this medical making spectrum, however few find a happy medium.

\subsection{The Right to Help and the Right Help}

Even though medical making carries greater risk than hobbyist activities, makers asserted their right to help. To an extent, every maker community in this study expressed a belief that they had a right to act: to make and deliver medical devices. Despite their altruistic intentions, there are consequences of unregulated medical making.

These communities leveraged technologies with minimal regulatory oversight. They often compromised on regulatory standards since they slow down their action-oriented efforts which rely on embodied, hands-on experience to harness material processes. Their efforts to help became a struggle to cope with regulations that exist to protect life, but are designed to be implemented by manufactures with extensive legal and compliance capabilities. In medical making, personal liability and collective accountability are often compromised with no recourse for device recipients. Unlike with traditional manufacturers there is no clear path to hold makers or maker communities accountable for the negative outcomes of their devices.

Regulation aims to push the locus of responsibility onto manufactures, but many makers viewed regulatory processes as obstructing their values of action and innovation. To slow the spread of incomplete, unverified, or unsafe designs, regulation prevents volunteer designers from releasing their work to be improved through selfcorrecting behavior in open source communities. This, in turn, delays community recognition of individual efforts and stalls the communal learning process (i.e., slowing design). These regulations become exacting as the inherent risks of a design increase (e.g., ventilators require more review than face shields). However, these more complex designs are valued amongst makers who often ascribe greater value to technical challenges than positive impact. In order to pursue the greater challenges they value more, some communities chose to operate in the highest-risk spaces (e.g., ventilators) while abandoning regulatory efforts altogether. When makers act as manufacturers but do not accept regulatory responsibilities, they force practitioners to bear the final consequences of faulty devices.

When these communities accept regulatory responsibilities in order to ensure safety, they rely on the forms of knowledge readily available to them. If principled knowledge is unavailable or is contradictory, makers may turn to health professionals because they view practical expertise as an acceptable substitute for principled knowledge. However, we note that medical practitioners, who know how to use a device (e.g., PPE), do not necessarily know how it functions; their experiences are not interchangeable. Practitioners rely on regulatory processes derived from principled knowledge to ensure that a device is safe and effective. Without access to a diverse set of knowledge (e.g., principled knowledge, practical knowledge, and embodied experiences), makers are at a disadvantage.
The resulting misalignment of principled knowledge and espoused practical expertise may be exacerbated by the rapid spread of antiregulatory sentiment and misinformation online during collective sense making. Communities that effectively limited the spread of misinformation tended to accept external regulation, while those that adopted conspiracy theories tended to internally regulate based on their own values.

Distaste for regulation does not confer the right or responsibility to act without caution. Even as supplies of traditional PPE ran dry, many hospitals continued to reject the products donated by makers. In some cases, makers pressured individual medical practitioners to accept and use their PPE despite administrative restrictions. We argue that the maker belief that they have a right to help can act as a barrier to provide the right help. Furthermore, makers who believe they can do enough good (e.g., if they can save one life with their ventilator while brushing aside risks for even one death) use this to justify their unregulated actions. The consequences of their trade-offs and risks are only visible to communities if there is a collective effort to identify them.

\subsection{Systems of Safety and Speed}

Makers' criticism of regulatory structures is not completely unfounded. Regulatory systems can slow down problem-solving, stunt multifarious design approaches, and raise the barrier for entry. Established systems of knowledge production and distribution are not designed for speedy action. Regulations favor centralized institutional settings with access to more resources. We recognize the justifiable demands of action-oriented makers to pursue unrestricted design and distribution. As a result, a tension arises between safety and speed and reveals opportunities for new ways of making and managing communities.

In normal circumstances, the public is not aware of the consensus forming debates that produce medical device regulations. However, research about the virus and the development of new PPE designs have occurred openly and concurrently, so non-technical makers could observe this sense making process in real time. It is in the public's interest to quickly disseminate and contextualize emergent knowledge. We observed community efforts to accomplish this in a context of their making with varying degrees of success. When the context remained unclear to makers, discourse broke down and action halted.

Without access to principled expertise, makers struggle to distill the requirements of medical devices regulation into actionable instructions. Decoding regulatory efforts to relax non-critical policies requires expertise beyond the capabilities of most makers, who are typically volunteers who lack domain knowledge. The NIH 3D Print Exchange attempted to create a pathway for medical makers to have their open source designs reviewed, but designers had little visibility into this process post-submission. As a result, communities chose to either offset the significant upfront cost of design work by distributing designs regardless of formal review or divert significant resources towards design and away from distribution. While some communities chose the latter, others distributed designs without clinical review which consumed finite resources (e.g., volunteer makers, materials) in faster, unregulated, and potentially harmful production. 
Despite these challenges, some communities persevered and followed external regulations, however even in these safety-focused communities tangible progress was critical. Otherwise, the momentum of maker activities is slowed as time is lost to serve redundant regulatory processes. In the end, strict adherence to a "do no harm" ethos prevented some communities from creating PPE. Striking the right balance between action and regulation is critical.

\section{DESIGN RECOMMENDATIONS}

Recognizing the spectrum between action and regulation is not sufficient for community self-determination of maker prerogatives. Community norms were also shaped by: confusion about regulatory processes, misinformation on online platforms, and content organization and dissemination. We make three design recommendations to strategically and purposefully position medical maker communities on this spectrum. First, we recommend that regulatory bodies foster relationships with maker communities. Second, we argue that community moderators should be given more control over content presentation. Third, we call for regulated repositories to be structured to inhibit the adoption of unverified, outdated, or poor-quality content.

\subsection{Prescriptive Regulatory Clarity}

A significant challenge in medical making communities is fostering trust between the community and regulatory systems. Some makers are likely to hold anti-regulatory beliefs, inherently opposed to the safety-centered impact of medical making activity. However, more makers may be amenable to collaborate and access adequate support when they can establish an understanding of the role and benefits of a regulatory structure. From our perspective, regulation has three key benefits. (1) Regulation is an external review process to improve device and procedure safety; ultimately, safety-focused communities will have more impact and sustainable results. (2) Regulatory agencies are useful networks of domain expertise, and can foster connections to medical practitioners and researchers; these relationships can improve designs rather than stalling them. (3) Regulated devices help establish trust with users, healthcare workers, leading to increased adoption of maker artifacts.

We attribute anti-regulatory sentiments, in part, to confusion about opaque regulatory practices. These are defined in complex legal language and structured around expensive, company-oriented processes. While these processes fit into the ecosystem of traditional medical manufacturing, they obstruct makers who tend to lack expert resources. The clinical review process within the NIH 3D Print Exchange is an effective first step, but connections to makers should be extended. The requirements of these review processes should be made explicit and put into simple language. Further, calls for specific needs that are amenable to consumer manufacturing should be directly pushed to these maker communities. Finally, communities need solidarity liaisons with regulatory systems to build trust, ask questions, and negotiate their hyper-local needs. We recommend that these liaisons be recruited from within government agencies, corporate partners, and university researchers. Makers will likely accept offered expertise, framed as a resource, particularly early in group formation. By establishing a norm of regulatory partnership, a culture of safety may be extended to more maker communities.

\subsection{Platform and Algorithmic Transparency}

Particularly in a pandemic which necessitates social distancing, virtual collaboration platforms were critical to enable collective medical making activities. However, many communities developed without clear insights into how the platforms' structure would influence their work. It is in the best interest of both the platforms and the communities to establish clear knowledge about how the algorithmic structure of these platforms directs the flow of content. For instance, posts matched with users and utterances can be, ideally, managed by community moderators, or, at least, made explicit to community members. If makers are directed away from specific posts or content, moderators need this information to ensure that critical knowledge is distributed to all community members. Conversely, members need granular control to filter the onslaught of community content especially during the shared environment of anxiety in a crisis. Slack's channeled structure, perhaps, provides a clue and could be improved with additional methods for crossposting information. The organizational effort of members can be offset by notifying specific information-silos of new, relevant content. Natural language processing may play a limited role in this, by identifying terms and phrases relevant to one user and periodically bringing in this content. Maker communities composed of fluid memberships have highly unique structures. Extending algorithmic support to community moderators requires the design of platforms to adapt their motivations.

\subsection{Extending Regulated Repository Infrastructure}

New information spread quickly, particularly in open source communities, as they reacted to the rapidly evolving crisis. As public knowledge develops and best practices are updated, dispersed repositories of community content may continue to spread outdated content or fall into disuse. We recommend that new repository infrastructures be developed to moderate and update the content that is dispersed to these communities. The NIH 3D Print Exchange presents a possible approach to this. However, the platform must extend beyond designs to include safety procedures, clinical review testing methodologies, and community development guidelines. Each influences the safety-driven practices of maker communities, and thus the quality of the devices they produce. Further, these may be presented in ways that generalize to other communities' designs. Makers build communal knowledge to disperse best practices that could benefit crisis response across disconnected communities. Such regulated repositories, require review guidelines to enable contributors a collaborative vision of medical making. While unreviewed content should be publicly available to encourage community iteration, the potential risks and consequences of using it should be made extensively explicit to prevent dissemination of potentially harmful content. Once reviewed iterations are available, past un-reviewed iterations or those that failed review should be immediately, ideally automatically, stripped from the repository to prevent confusion between the outdated and updated versions. 


\section{LIMITATIONS AND ETHICS}

As researchers with close relations to medical practitioners, we personally espouse a "do no harm" ethos. As a result, we may have more favorable views of communities and makers that emphasize safety. Additionally, as researchers in medical making prior to the onset of the pandemic, we felt an ethical responsibility to participate in these efforts. As such, conducting participatory observations was not just a methodological decision, but an ethical choice which enabled us to apply our expertise where we believed it was applicable. On the spectrum between action-oriented making and regulated making, we would position ourselves as researchers closer to regulation, with a cautiously optimistic view of the role of making as repair of medical infrastructure. As participant observers, we focused on communities within our reach. So even though we found that medical making is a global effort, our findings are rooted in the medical, legal, and technical infrastructure of the United States.

\section{CONCLUSION}

Prior to the COVID-19 pandemic, medical making was relatively rare, but by mid-March 2020 medical maker communities were quickly forming to meet the rising tide of PPE demand. In a rapidly changing medical and regulatory environment, medical maker communities developed community norms, guided by their beliefs in expertise and safety, which informed their practices. In this paper, we present the results of a participant-observation study of 14 medical-maker communities conducted between March and June of 2020 .

Based on this study, we presented three themes. We found that: (1) communities tend to be either action-oriented, safety-driven, or design-driven; (2) communities tend to prioritize either principled knowledge, professional practice, or embodied experiences; and (3) features of online communication platform could inhibit community planning, access to established procedures, and constructive community engagement. Overall, these three distinguishing features of communities position them on a spectrum between internally- and externally- regulated efforts.

We argue for concerted efforts to adapt infrastructure and recognize its role in shaping these emergent medical making communities. Our recommendations encourage communities to intentionally position themselves with support from: (1) regulatory bodies' policies that foster stronger coalitions with medical makers; (2) online community platforms that enable moderators to more closely control how content is presented based on the communities' needs; and (3) medical making repositories that expand their collections to include safety procedures and methodologies while limiting the spread of incomplete or unverified designs. By building a more robust medical making infrastructure rooted in the practices of maker communities, we expect that communities could thrive as an alternate mechanism for care work by a diverse group of global communities to safely design, produce, and disseminate medical devices in a timely manner that meet local needs.

\section{ACKNOWLEDGMENTS}

This work would not be possible without the openness of the communities we studied. This work was funded by the National Science Foundation Grants: IIS-1718651, IIS-1907337, 1836813, and 2031801 and supported by UW Create.

\section{REFERENCES}

[1] Daniel Auferbauer and Hilda Tellioğlu. 2019. Socio-Technical Dynamics: Cooperation of Emergent and Established Organisations in Crises and Disasters. In Proceedings of the 2019 CHI Conference on Human Factors in Computing Systems (Glasgow, Scotland Uk) (CHI '19). Association for Computing Machinery, New York, NY, USA, 1-13. https://doi.org/10.1145/3290605.3300448

[2] Geoff Baker. 2020. As 3D printing of protective gear ramps up, a free mask designed in Seattle is the first of its kind to get federal approval. https://www.seattletimes.com/business/as-3d-printing-of-protective-gearramps-up-a-free-mask-designed-in-seattle-is-the-first-of-its-kind-to-getfederal-approval/

[3] Erin Buehler, Stacy Branham, Abdullah Ali, Jeremy J. Chang, Megan Kelly Hofmann, Amy Hurst, and Shaun K. Kane. 2015. Sharing is Caring: Assistive Technology Designs on Thingiverse. In Proceedings of the 33rd Annual ACM Conference on Human Factors in Computing Systems (CHI '15). ACM, New York, NY, USA, 525-534. https://doi.org/10.1145/2702123.2702525

[4] Erin Buehler, Niara Comrie, Megan Hofmann, Samantha McDonald, and Amy Hurst. 2016. Investigating the Implications of 3D Printing in Special Education. ACM Trans. Access. Comput. 8, 3 (March 2016), 11:1-11:28. https://doi.org/10. $1145 / 2870640$

[5] Guy Burdick. 2020. COVID-19 Crisis Puts Spotlight on PPE at Healthcare Facilities. https://www.psqh.com/news/covid-19-crisis-puts-spotlight-on-ppe-athealthcare-facilities/

[6] John W. Creswell and Cheryl N. Poth. 2016. Qualitative Inquiry and Research Design: Choosing Among Five Approaches. SAGE Publications, 2455 Teller Rd, Thousand Oaks, CA 91320. Google-Books-ID: DLbBDQAAQBAJ.

[7] Laura Dabbish, Colleen Stuart, Jason Tsay, and Jim Herbsleb. 2012. Social Coding in GitHub: Transparency and Collaboration in an Open Software Repository. In Proceedings of the ACM 2012 Conference on Computer Supported Cooperative Work (Seattle, Washington, USA) (CSCW'12). Association for Computing Machinery, New York, NY, USA, 1277-1286. https://doi.org/10.1145/2145204.2145396

[8] Williams DeClark. 2018. Who is Liable for a Defective 3D Printed Medical Device? https://www.wdtlaw.org/personal-injury-blog/2018/february/who-isliable-for-a-defective-3d-printed-medical/

[9] Bryan Dosono and Bryan Semaan. 2019. Moderation Practices as Emotional Labor in Sustaining Online Communities: The Case of AAPI Identity Work on Reddit. In Proceedings of the 2019 CHI Conference on Human Factors in Computing Systems (Glasgow, Scotland Uk) (CHI '19). Association for Computing Machinery, New York, NY, USA, 1-13. https://doi.org/10.1145/3290605.3300372

[10] Hayley I. Evans, Marisol Wong-Villacres, Daniel Castro, Eric Gilbert, Rosa I. Arriaga, Michaelanne Dye, and Amy Bruckman. 2018. Facebook in Venezuela: Understanding Solidarity Economies in Low-Trust Environments. In Proceedings of the 2018 CHI Conference on Human Factors in Computing Systems (Montreal QC, Canada) (CHI '18). Association for Computing Machinery, New York, NY, USA, 1-12. https://doi.org/10.1145/3173574.3173802

[11] Frederika Farley. 1938. Improvising Equipment. Am. F. Nurs. 38, 4 (Apr 1938), 42s-43s. http://www.jstor.org/stable/3414253

[12] Casey Fiesler and Amy S. Bruckman. 2019. Creativity, Copyright, and CloseKnit Communities: A Case Study of Social Norm Formation and Enforcement. Proc. ACM Hum.-Comput. Interact. 3, GROUP, Article 241 (Dec. 2019), 24 pages. https://doi.org/10.1145/3361122

[13] Sarah Fox, Rachel Rose Ulgado, and Daniela Rosner. 2015. Hacking Culture, Not Devices: Access and Recognition in Feminist Hackerspaces. In Proceedings of the 18th ACM Conference on Computer Supported Cooperative Work \& Social Computing (Vancouver, BC, Canada) (CSCW'15). Association for Computing Machinery, New York, NY, USA, 56-68. https://doi.org/10.1145/2675133.2675223

[14] Jose Gomez-Marquez and Anna Young. 2016. A History of Nurse Making and Stealth Innovation. https://doi.org/10.2139/ssrn.2778663 [Online; accessed 21. Dec. 2020].

[15] Megan Hofmann, Julie Burke, Jon Pearlman, Goeran Fiedler, Andrea Hess, Jon Schull, Scott E. Hudson, and Jennifer Mankoff. 2016. Clinical and Maker Perspectives on the Design of Assistive Technology with Rapid Prototyping Technologies. In Proceedings of the 18th International ACM SIGACCESS Conference on Computers and Accessibility (ASSETS '16). ACM, New York, NY, USA, 251-256. https://doi.org/10.1145/2982142.2982181

[16] Megan Hofmann, Kristin Williams, Toni Kaplan, Stephanie Valencia, Gabriella Hann, Scott E. Hudson, Jennifer Mankoff, and Patrick Carrington. 2019. "Occupational Therapy is Making": Clinical Rapid Prototyping and Digital Fabrication. In Proceedings of the 2019 CHI Conference on Human Factors in Computing Systems (Glasgow, Scotland Uk) (CHI '19). Association for Computing Machinery, New York, NY, USA, 1-13. https://doi.org/10.1145/3290605.3300544

[17] Y. Linlin Huang, Kate Starbird, Mania Orand, Stephanie A. Stanek, and Heather T. Pedersen. 2015. Connected Through Crisis: Emotional Proximity and the Spread of Misinformation Online. In Proceedings of the 18th ACM Conference on Computer Supported Cooperative Work \& Social Computing (Vancouver, BC, Canada) (CSCW 
'15). Association for Computing Machinery, New York, NY, USA, 969-980. https: //doi.org/10.1145/2675133.2675202

[18] Darrell Hurt, Nick Weber, and Meghan Coakley McCarthy. 2019. About the NIH 3D Print Exchange $\mid$ NIH 3D Print Exchange. https://3dprint.nih.gov/about

[19] Jonathan P. Jarow and John H. Baxley. 2015. Medical devices: US medical device regulation. Urologic Oncology 33, 3 (March 2015), 128-132. https://doi.org/10. 1016/j.urolonc.2014.10.004

[20] Quentin Jones, Gilad Ravid, and Sheizaf Rafaeli. 2004. Information Overload and the Message Dynamics of Online Interaction Spaces: A Theoretical Model and Empirical Exploration. Info. Sys. Research 15, 2 (June 2004), 194-210. https: //doi.org/10.1287/isre.1040.0023

[21] Charles Kiene, Andrés Monroy-Hernández, and Benjamin Mako Hill. 2016. Surviving an "Eternal September": How an Online Community Managed a Surge of Newcomers. In Proceedings of the 2016 CHI Conference on Human Factors in Computing Systems (San Jose, California, USA) (CHI '16). Association for Computing Machinery, New York, NY, USA, 1152-1156. https://doi.org/10.1145/2858036.2858356

[22] Robert E. Kraut, Paul Resnick, Sara Kiesler, Yuqing Ren, Yan Chen, Moira Burke, Niki Kittur, John Riedl, and Joseph Konstan. 2012. Building Successful Online Communities: Evidence-Based Social Design. The MIT Press, 1 Rogers St, Cambridge, MA 02142

[23] Stacey Kuznetsov and Eric Paulos. 2010. Rise of the Expert Amateur: DIY Projects, Communities, and Cultures. In Proceedings of the 6th Nordic Conference on HumanComputer Interaction: Extending Boundaries (Reykjavik, Iceland) (NordiCHI '10). ACM, New York, NY, USA, 295-304. https://doi.org/10.1145/1868914.1868950

[24] Udaya Lakshmi, Megan Hofmann, Stephanie Valencia, Lauren Wilcox, Jennifer Mankoff, and Rosa I. Arriaga. 2019. "Point-of-Care Manufacturing": Maker Perspectives on Digital Fabrication in Medical Practice. Proc. ACM Hum.-Comput. Interact. 3, CSCW, Article 91 (Nov. 2019), 23 pages. https://doi.org/10.1145/ 3359193

[25] Yvonna S Lincoln and Egon G Guba. 1985. Naturalistic inquiry (vol. 75).

[26] Silvia Lindtner, Anna Greenspan, and David Li. 2015. Designed in Shenzhen $<\mathrm{i}>$ Shanzhai</i> Manufacturing and Maker Entrepreneurs. In Proceedings of The Fifth Decennial Aarhus Conference on Critical Alternatives (Aarhus, Denmark) (CA '15). Aarhus University Press, Aarhus N, 85-96. https://doi.org/10.7146/ aahcc.v1i1.21265

[27] Silvia Lindtner, Garnet D. Hertz, and Paul Dourish. 2014. Emerging Sites of HCI Innovation: Hackerspaces, Hardware Startups Incubators. In Proceedings of the SIGCHI Conference on Human Factors in Computing Systems (Toronto, Ontario, Canada) (CHI '14). ACM, New York, NY, USA, 439-448. https://doi.org/10.1145/ 2556288.2557132

[28] German Lopez. 2020. Why America ran out of protective masks - and what can be done about it. https://www.vox.com/policy-and-politics/2020/3/27/21194402/ coronavirus-masks-n95-respirators-personal-protective-equipment-ppe

[29] Malpractice 2020. What is Malpractice? https://www.abpla.org/what-ismalpractice

[30] David R. Marshall and Deborah A. McGrew. 2017. Creativity and Innovation in Health Care: Opening a Hospital Makerspace. Nurse Leader 15, 1 (2017), 56 - 58 https://doi.org/10.1016/j.mnl.2016.10.002

[31] Samantha McDonald, Niara Comrie, Erin Buehler, Nicholas Carter, Braxton Dubin, Karen Gordes, Sandy McCombe-Waller, and Amy Hurst. 2016. Uncovering Challenges and Opportunities for 3D Printing Assistive Technology with Physical Therapists. In Proceedings of the 18th International ACM SIGACCESS Conference on Computers and Accessibility (Reno, Nevada, USA) (ASSETS '16). Association for Computing Machinery, New York, NY, USA, 131-139. https://doi.org/10. 1145/2982142.2982162

[32] Caitlin McLaughlin and Jessica Vitak. 2012. Norm evolution and violation on Facebook. New Media \& Society 14, 2 (March 2012), 299-315. https://doi.org/10. 1177/1461444811412712 Publisher: SAGE Publications.

[33] Nightscout 2020. Welcome to Nightscout. http://www.nightscout.info/

[34] NIH COVID-19 Response 2020. COVID-19 Response | NIH 3D Print Exchange. https://3dprint.nih.gov/collections/covid-19-response

[35] Michael H. Park. 2015. For a New Heart, Just Click Print: The Effect on Medical and Products Liability from 3-D Printed Organs Notes. University of Illinois Journal of Law, Technology \& Policy 2015 (2015), 187-210.

[36] Jeremiah Parry-Hill, Patrick C. Shih, Jennifer Mankoff, and Daniel Ashbrook. 2017. Understanding Volunteer AT Fabricators: Opportunities and Challenges in DIY-AT for Others in e-NABLE. In Proceedings of the 2017 CHI Conference on Human Factors in Computing Systems (Denver, Colorado, USA) (CHI '17). Association for Computing Machinery, New York, NY, USA, 6184-6194. https: //doi.org/10.1145/3025453.3026045

[37] David Roedl, Shaowen Bardzell, and Jeffrey Bardzell. 2015. Sustainable Making? Balancing Optimism and Criticism in HCI Discourse. ACM Trans. Comput.-Hum. Interact. 22, 3, Article 15 (June 2015), 27 pages. https://doi.org/10.1145/2699742

[38] Clare Scott. 2018. 3D Printed, Open Source Glia Stethoscope Receives Clinical Validation - 3DPrint.com | The Voice of 3D Printing / Additive Manufacturing. https://3dprint.com/206934/glia-stethoscope-validation/

[39] Bryan Semaan and Gloria Mark. 2011. Technology-Mediated Social Arrangements to Resolve Breakdowns in Infrastructure during Ongoing Disruption. ACM Trans. Comput.-Hum. Interact. 18, 4, Article 21 (Dec. 2011), 21 pages. https: //doi.org/10.1145/2063231.2063235

[40] John T. Sherrill. 2016. Teaching Documentation through 3D Printing and Instructables. In Proceedings of the 34th ACM International Conference on the Design of Communication (Silver Spring, MD, USA) (SIGDOC '16). Association for Computing Machinery, New York, NY, USA, Article 56, 1 pages. https: //doi.org/10.1145/2987592.2987652

[41] Karin Slegers, Kristel Kouwenberg, Tereza Loučova, and Ramon Daniels. 2020. Makers in Healthcare: The Role of Occupational Therapists in the Design of DIY Assistive Technology. In Proceedings of the 2020 CHI Conference on Human Factors in Computing Systems (Honolulu, HI, USA) (CHI '20). Association for Computing Machinery, New York, NY, USA, 1-11. https://doi.org/10.1145/3313831.3376685

[42] Kate Starbird, Ahmer Arif, and Tom Wilson. 2019. Disinformation as Collaborative Work: Surfacing the Participatory Nature of Strategic Information Operations. Proc. ACM Hum.-Comput. Interact. 3, CSCW, Article 127 (Nov. 2019), 26 pages. https://doi.org/10.1145/3359229

[43] Kate Starbird, Dharma Dailey, Owla Mohamed, Gina Lee, and Emma S. Spiro. 2018. Engage Early, Correct More: How Journalists Participate in False Rumors Online during Crisis Events. In Proceedings of the 2018 CHI Conference on Human Factors in Computing Systems (Montreal QC, Canada) (CHI '18). Association for Computing Machinery, New York, NY, USA, 1-12. https://doi.org/10.1145/ 3173574.3173679

[44] Jeannette N Sutton, Leysia Palen, and Irina Shklovski. 2008. Backchannels on the front lines: Emergency uses of social media in the 2007 Southern California Wildfires. (2008)

[45] Joshua G. Tanenbaum, Amanda M. Williams, Audrey Desjardins, and Karen Tanenbaum. 2013. Democratizing Technology: Pleasure, Utility and Expressiveness in DIY and Maker Practice. In Proceedings of the SIGCHI Conference on Human Factors in Computing Systems (Paris, France) (CHI '13). ACM, New York, NY, USA, 2603-2612. https://doi.org/10.1145/2470654.2481360

[46] Cristen Torrey, Moira Burke, Matthew Lee, Anind Dey, Susan Fussell, and Sara Kiesler. 2007. Connected Giving: Ordinary People Coordinating Disaster Relief on the Internet. In Proceedings of the 40th Annual Hawaii International Conference on System Sciences (HICSS '07). IEEE Computer Society, USA, 179a. https://doi. org/10.1109/HICSS.2007.144

[47] Arifumi Utani, Teruhiro Mizumoto, and Takashi Okumura. 2011. How Geeks Responded to a Catastrophic Disaster of a High-Tech Country: Rapid Development of Counter-Disaster Systems for the Great East Japan Earthquake of March 2011. In Proceedings of the Special Workshop on Internet and Disasters (Tokyo, Japan) (SWID '11). Association for Computing Machinery, New York, NY, USA, Article 9,8 pages. https://doi.org/10.1145/2079360.2079369

[48] Dhaval Vyas. 2019. Altruism and Wellbeing as Care Work in a Craft-Based Maker Culture. Proc. ACM Hum.-Comput. Interact. 3, GROUP, Article 239 (Dec. 2019), 12 pages. https://doi.org/10.1145/3361120

[49] Tara Whelan. 2018. We Are Not All Makers: The Paradox of Plurality In The Maker Movement. In Proceedings of the 2018 ACM Conference Companion Publication on Designing Interactive Systems (Hong Kong, China) (DIS '18 Companion). Association for Computing Machinery, New York, NY, USA, 75-80. https://doi.org/10.1145/3197391.3205415

[50] Marisol Wong-Villacres, Cristina M. Velasquez, and Neha Kumar. 2017. Social Media for Earthquake Response: Unpacking Its Limitations with Care. Proc. ACM Hum.-Comput. Interact. 1, CSCW, Article 112 (Dec. 2017), 22 pages. https: //doi.org/10.1145/3134747

[51] Anna Young. 2015. How the DIY Mindset Can Help Us Build a Culture of Health. http://blog.tedmed.com/how-the-diy-mindset-can-help-us-build-aculture-of-health/ 